\title{
Candidate Biomarkers for the Diagnosis of Transient Ischemic Attack: A Systematic Review
}

\author{
L. Servaas Dolmans ${ }^{\mathrm{a}}$ Frans H. Rutten ${ }^{\mathrm{a}}$ Niels C.T. Koenen ${ }^{\mathrm{a}}$ \\ Marie-Louise E.L. Bartelink ${ }^{\mathrm{a}} \quad$ Johannes B. Reitsma ${ }^{\mathrm{a}} \quad$ L. Jaap Kappelle ${ }^{\mathrm{b}}$
}

Arno W. Hoes ${ }^{\text {a }}$

a Julius Center for Health Sciences and Primary Care, University Medical Center, Utrecht University, Utrecht, The Netherlands; ${ }^{b}$ Department of Neurology, University Medical Center, Utrecht University, Utrecht, The Netherlands

\section{Keywords}

Transient ischemic attack · Biomarker · Diagnosis · Review

\begin{abstract}
Background and Purpose: A rapid serum biomarker that confirms or rules out a transient ischemic attack (TIA) would be of great value in clinical practice. We aimed to systematically review current evidence for the diagnostic accuracy of blood biomarkers in the early diagnosis of TIA. Methods: This is a systematic review with quality appraisal of individual studies using the QUADAS-2 tool. MEDLINE and EMBASE databases were searched up to May 1, 2017, to select primary diagnostic accuracy studies evaluating potential biomarkers in blood for the diagnosis of TIA or ischemic stroke. Results: Of 4,215 studies retrieved, 78 met our eligibility criteria. Forty-five studies restricted their population to ischemic stroke patients, 32 included both TIA and ischemic stroke patients, and only one study was restricted to TIA patients. In total $62 / 78(79.5 \%)$ studies had a case-control design comparing TIA or stroke patients with healthy subjects. Overall, 125 single biomarkers and 5 biomarker panels were studied, with a median number of participants per study of 92.0 (interquartile range 44.8-144.5), varying from 8 to 915 . Sufficient information to extract $2 \times 2$ tables was available for 35 (44.9\%)
\end{abstract}

articles, and for 60 (48.0\%) biomarkers. Several markers, such as NR2A/B (antibodies), Parkinson 7, nucleoside diphosphate kinase $A$, ubiquitin fusion degradation protein-1, and heart-type fatty acid binding protein, have shown moderate to high diagnostic accuracy in multiple studies. Conclusions: Although the methodological quality of studies evaluating biomarkers of brain ischemia was poor, several biomarkers have shown the potential to detect transient brain ischemia in an early phase. Diagnostic accuracy studies in suspected cases of TIA are needed to determine their true clinical value.

(c) 2019 The Author(s) Published by S. Karger AG, Basel

\section{Introduction}

The clinical diagnosis of transient ischemic attack (TIA) can be difficult for both the general practitioner and neurologist. Timely recognition of TIA is important, since the risk of a subsequent ischemic stroke is especially high in the first days after a TIA and early initiation of treatment substantially reduces this risk $[1,2]$. A rapid serum biomarker that confirms or rules out TIA would be of great value in clinical practice.

L. Servaas Dolmans, MD

Julius Center for Health Sciences and Primary Care

University Medical Center Utrecht, Utrecht University

Stratenum 6.131, NL-3508 GA Utrecht (The Netherlands)

E-Mail L.S.Dolmans@umcutrecht.nl 
To date, the diagnosis of TIA still mainly relies on precise history taking. The initial evaluation of patients suspected of TIA is often performed by a general practitioner, without further additional testing at that point. Establishing or excluding TIA can be difficult for several reasons. TIAs may present atypically, the symptoms are often short-lasting and resolved at consultation, and there is a broad differential diagnosis, for example, migraine, seizures (pre)syncope, and vestibular syndromes. An early detectable biomarker could help clinicians to diagnose TIA more accurately within a shorter time frame. Rapid and correct exclusion of TIA would save costly referrals to a TIA outpatient clinic, while confirmation of TIA facilitates early (anti-thrombotic) treatment and can reduce the risk of subsequent stroke.

A rapidly growing range of biomarkers associated with brain ischemia has been tested, especially in patients with a possible stroke for purposes of diagnosis, and early prognosis. Yet, no diagnostic biomarkers are used in everyday clinical practice for detection or exclusion of TIA or stroke. Previous reviews on stroke biomarkers emphasized the difficulties concerning biodynamic aspects ( such as the influence of the specific region of ischemia and the role of the blood-brain barrier delaying the release of proteins) and the methodological limitations of biomarker studies $[3,4]$.

TIA and ischemic stroke must be regarded as a continuum, both initiating the same ischemic cascade, but with a different level of severity. Biomarkers of TIA reflect this ischemic cascade and not (necessarily) cell necrosis. Because of the difference in degree of brain ischemia, biomarker values will often be lower and closer to normal values in TIA patients than in stroke patients. Moreover, because in TIA symptoms and signs relieve fast, there is often more delay in seeking medical attention by patients with a TIA than in the case of severe and/or persisting clinical features as in stroke.

A useful diagnostic biomarker for TIA must first of all be sensitive to low grades of ischemia, and detectable in blood from the first hour till several days after symptoms. Furthermore, the biomarker must differentiate TIAs from a heterogeneous group of alternative diagnoses to be of use in clinical practice. To evaluate clinical relevance, diagnostic accuracy studies among suspected patients are needed, as opposed to studies that compare cases with healthy subjects [5]. These latter studies are a logical first step in the evaluation of new markers that provide a sense of their potential value, but typically overestimate the diagnostic performance when measured in suspected patients in whom the markers will be used in practice.

We aimed to systematically review current evidence for the use of blood biomarkers in the early diagnosis of TIA.

\section{Methods}

A literature search was conducted following PRISMA guidelines and using the MEDLINE and EMBASE databases, last updated May 1, 2017 [6]. We used the key terms shown in Box 1 to find papers evaluating potential biochemical markers for the diagnosis of TIA. Although our actual domain of interest was patients with transient symptoms suspected of TIA, we broadened our search to the whole spectrum of brain ischemia, instead of restricting to TIA only, as a pilot search showed that most published studies tested biomarkers in a population with both ischemic stroke and TIA cases. To narrow our search to diagnostic studies we used a set of diagnostic terms.

Two reviewers screened titles and abstracts for relevance (L.S.D. and N.C.T.K.). A first sample of articles was used to cross-check the selection process. Full texts of selected articles were reviewed independently by both reviewers. Primary studies on the diagnostic value of blood biomarkers in patients with (or suspected of) TIA or ischemic stroke were included. Animal studies, prognostic studies, conference abstracts, and non-English publications were excluded. We also screened reference lists of included articles.

Data were extracted with a standardized data extraction form, which we included as supplementary file. The quality of included studies was assessed with the modified QUADAS-2 tool [7]. Disagreements between the 2 reviewers were resolved by discussion. The most important aspects of data extraction were:

- Relevance to clinical domain: (to what extent) is the biomarker tested in TIA (instead of stroke) patients? Most relevant to our domain is a study population of patients suspected of TIA, as opposed to studies using a case-control design.

- Timing of blood sampling: is it reported and does it match an early diagnosis of TIA, that is, the usual time window of diagnostic assessment is from the same day up to several days after the event?

- Adequate reference standard: diagnostic assessment by a neurologist with the use of neuroimaging was the minimum requirement. Ideally a panel of neurologists using such information and detailed history taking represented the reference standard [8].

- Relevant measures of diagnostic accuracy: is a cut-off used and was it pre-defined? Most relevant measures considered were predictive values calculated from a $2 \times 2$ table in univariate anal$y$ sis, and ORs and the area under the receiver operating characteristics curve (AUC) or C-statistic in multivariate analysis. Ideally the added value of a biomarker was calculated in addition to relevant items of history taking or clinical judgment, and results were validated in a second group of suspected patients.

\section{Definition of TIA}

In the data extraction, we also assessed the applied definitions of TIA and minor stroke. The original time-based definition of TIA is based on a maximum duration of symptoms of $24 \mathrm{~h}$. The new tissue-based definition of TIA was introduced in 2009 following advancements in neuroimaging techniques and includes the criterion of absence of infarction on brain imaging [9]. Around $30-40 \%$ of those classified as TIA with the old definition would be classified as minor stroke with the new definition, when using high resolution MRI [10, 11]. Currently, the tissue-based definition is most widely endorsed because differentiating minor strokes yields prognostic information. However, the time-based definition is still 
Box 1. Search terms used

PubMed search terms

$((((((()((($ biological markers[MeSH Terms] $)$ OR diagnostic marker*[Title/Abstract] $)$ OR biomarker[Title/Abstract]) OR marker*[Title/Abstract]) OR antibody[Title/Abstract]) OR antibodies[Title/Abstract]) OR antigen*[Title/Abstract]) OR laboratory test[Title/Abstract]) OR blood test[Title/Abstract]) OR *RNA[Title/Abstract]) OR microRNA[Title/Abstract])) AND

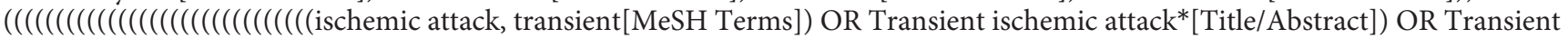
ischaemic attack*[Title/Abstract]) OR TIA[Title/Abstract]) OR TIAs[Title/Abstract]) OR attack* AND, transient ischemic[Title/ Abstract]) OR attack* AND , transient ischaemic[Title/Abstract]) OR Ischemic attack* AND, transient[Title/Abstract]) OR ischaemic attack* AND, transient[Title/Abstract]) OR cerebral ischemia*[Title/Abstract]) OR cerebral ischaemia*[Title/Abstract]) OR brain ischemia*[Title/Abstract]) OR brain ischaemia*[Title/Abstract]) OR Stroke*[Title/Abstract]) OR stroke[MeSH Terms]) OR CVA[Title/Abstract]) OR CVAs[Title/Abstract]) OR Cerebrovascular accident*[Title/Abstract]) OR brain vascular accident*[Title/Abstract]) OR vascular accident* AND, brain[Title/Abstract]) OR brain infarction[Title/Abstract]) OR cerebral infarction[Title/Abstract]) OR ischemic brain[Title/Abstract]) OR ischaemic brain[Title/Abstract]) OR ischemic neuronal[Title/ Abstract]) OR ischaemic neuronal[Title/Abstract]) OR neuronal ischemia[Title/Abstract]) OR neuronal ischaemia[Title/ Abstract]) OR ischemic encephalopathy[Title/Abstract]) OR ischaemic encephalopathy[Title/Abstract])) AND (((((()((sens[Title/ Abstract]) OR spec[Title/Abstract]) OR sensitiv*[Title/Abstract]) OR specific*[Title/Abstract]) OR diagno*[Title/Abstract]) OR area[Title/Abstract]) OR auc[Title/Abstract]) OR roc[Title/Abstract]) OR false[Title/Abstract])

Animal studies filtered out

Embase search terms

("biological marker"/exp OR "biochemical marker"/exp OR “molecular marker"/exp OR biomarker*:ab,ti OR "laboratory test”: ab,ti OR “laboratory tests”:ab,ti OR “blood test”:ab,ti OR “blood tests”:ab,ti OR rna:ab,ti OR microrna:ab,ti) AND (“brain infarction"/exp OR “brain ischemia”/exp OR “cerebrovascular accident”/exp OR “transient ischemic attack":ab,ti OR "transient ischaemic attack":ab,ti OR tia:ab,ti OR tias:ab,ti OR “cerebral ischemia”:ab,ti OR “cerebral ischaemia":ab,ti OR "brain ischaemia”: ab,ti OR stroke*:ab,ti OR cva:ab,ti OR cvas:ab,ti OR “cerebrovascular accidents”:ab,ti OR “cerebral infarction”:ab,ti OR “ischemic brain":ab,ti OR “ischaemic brain":ab,ti OR "ischemic encephalopathy":ab,ti OR "ischaemic encephalopathy":ab,ti) AND (sens:ab,ti OR spec:ab,ti OR sensitiv*:ab,ti OR specific*:ab,ti OR diagno*:ab,ti OR area:ab,ti OR auc:ab,ti OR roc:ab,ti OR false:ab,ti) AND [humans]/lim AND [embase]/lim

often being used by neurologists and researchers, certainly when a high resolution MRI scan is not routinely available in the clinical setting.

\section{Estimation of AUC}

Many studies did not report measures of diagnostic accuracy. To estimate the discriminative ability of the markers in these studies, we used methods to derive an AUC from reported (absolute) biomarker values. From the mean values and SD of diseased (i.e., TIA/stroke) and non-diseased patients, an AUC can be estimated [12]. If medians and interquartile ranges were reported, we first converted these into means and SD by using a log normal distribution. We used the approach of Hanley and McNeil to compute a CI for the AUC, based on the number of diseased and non-diseased cases [13]. The latter was also done for studies that reported an AUC without CI. In this way, we were able to give an illustrative overview of both reported and estimated AUCs for different markers in a forest plot.

\section{Data Synthesis}

Because of the expected heterogeneity of the results we did not aim for data pooling or meta-analysis. First, we will present an overview of the quality assessments of the identified studies, and the total number of biomarkers evaluated. Second, study results of individual potential biomarkers will be described. This concerns a selection of markers that best comply with our clinical domain and the criteria described above, thus based on a combination of ap-

Candidate Biomarkers for the Diagnosis

of TIA: A Systematic Review plicability, methodological quality of (current) evidence, and the diagnostic accuracy of markers. Because of the limited number of studies with clinical populations of patients suspected of TIA (or stroke), we also (shortly) discuss studies that used a case-control design comparing TIA (or stroke) patients with healthy subjects (not suspected of TIA).

\section{Results}

Our search identified a total of 4,215 studies. A flowchart of the review process is supplemented as appendix (Fig. 1). All abstracts were read, 198 articles were read in full, and 78 studies met our eligibility criteria.

These studies included a total of 17,216 participants, of which 9,391 (54.5\%) were patients diagnosed with a cerebrovascular event, and 7,825 (45.5\%) were either patients with stroke mimicking diagnoses $(n=1,399)$ or "healthy" volunteers $(n=6,426)$. The total number of patients with a TIA was 1,141 (12.1\% of 9,391 cerebrovascular events). The median number of TIA or stroke patients per study was 92.0 (interquartile range 44.8-144.5), varying from 8 to 915 patients. 
Fig. 1. Flowchart of the systematic review search strategy. TIA, transient ischemic attack; CSF, cerebrospinal fluid.

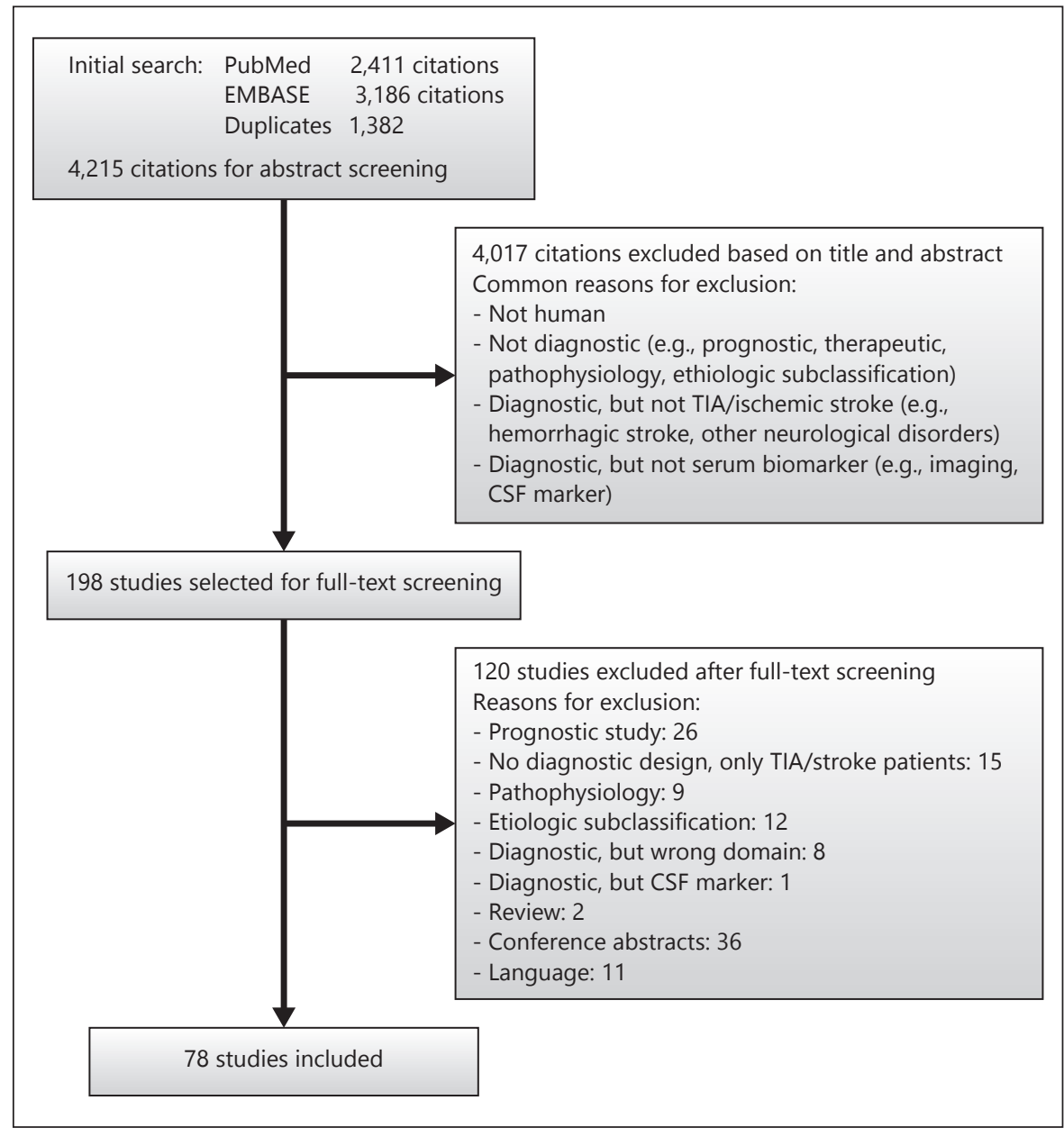

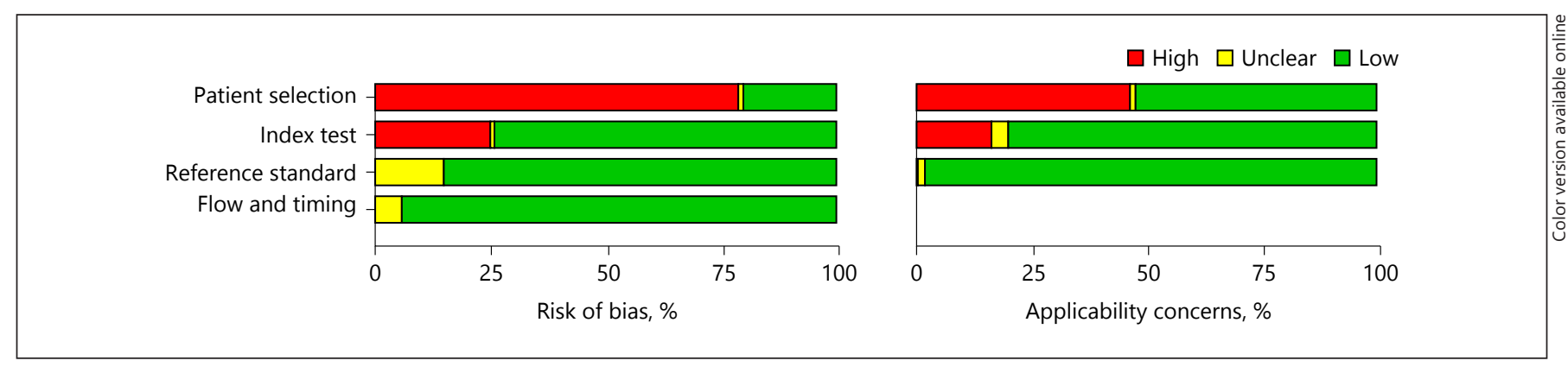

Fig. 2. Risk of bias and applicability concerns summary about each QUADAS-2 domain presented as percentages across the 78 included studies.

\section{Quality Assessment}

An overview of the results of the (modified) QUADAS-2 assessment is given in Figure 2. None of the studies restricted the study population to suspected TIA patients only. One study (1.3\%) included only established TIAs in a case-control design, comparing cases with TIA with healthy volunteers as controls [14]. In 45 (57.7\%) studies, biomarkers were examined in stroke patients only, excluding patients with TIA. In the remaining 32 (41.0\%) studies, the population was mixed, with both TIA and stroke patients. Patients suspected of stroke or TIA were included in 16 (20.5\%) studies. 
A predefined cut-off value was mentioned in 4 (5.1\%) studies. In 37 (47.4\%) studies, an optimal cut-off was derived from the examined cohort, of which 3 (8.1\%) studies were externally validated in a different cohort. Twenty-three (29.5\%) studies compared mean values of the biomarkers between those who eventually showed to have a stroke or TIA versus those without stroke or TIA.

Most studies used the evaluation of the attending neurologist as the reference standard for the diagnosis of TIA or stroke. In 5 (6.4\%) studies, a panel diagnosis was used (2 or 3 panel members). Classification of ischemic cerebrovascular disease according to the tissue-based definition of TIA was reported in only one study. The remaining studies all used the time-based definition for TIA or did not report the applied definition of TIA. Some studies (12 of 32 [37.5\%]) clearly distinguish TIA from stroke as a separate entity in their main analysis. Three studies $(9.4 \%)$ classified TIA as a non-stroke diagnosis. Most often (17 of 32 [53.1\%]) TIA and (minor) stroke were combined as ischemic cerebrovascular diagnoses.

Most studies described the time window of blood sampling for biomarker assessment in relation to the initiation of symptom or signs, 17 (21.8\%) studies reported the actual time (median or mean) to blood sampling.

\section{Biomarkers Identified}

A total of 124 single biomarkers and 5 biomarker panels were studied. Of the single biomarkers, 91 (73.4\%) were only evaluated in a case-control design, of which 74 in a single study and 17 in 2 or more studies. The remaining 33 (26.6\%) markers were evaluated in at least one study among patients suspected of TIA or stroke, of which 24 markers were evaluated in multiple studies (including case-control studies). The number of biomarkers tested per study varies from 1 to 17 . Sufficient information to extract $2 \times 2$ tables was reported in 35 $(44.9 \%)$ articles, and for $60(48.4 \%)$ biomarkers. Of these markers, only one combination of 4 markers was examined in a second cohort using the same cut-off [15]. Of the biomarker panels, one was evaluated among suspected cases $[16,17]$, and 4 in a single case-control study.

In the next section, we will discuss results of several candidate biomarkers that showed potential to be an early marker of TIA. Figure 3 presents a forest plot with both reported and estimated AUCs per study of these markers. Additionally, a scoping plot (Fig. 4) depicts to what extent the same markers have been evaluated in the domain of interest, that is, patients suspected of TIA.

Candidate Biomarkers for the Diagnosis

of TIA: A Systematic Review

\section{Individual Biomarkers}

NR2A/2B and NR2A/2B Antibodies

$\mathrm{NR} 2 \mathrm{~A} / 2 \mathrm{~B}$ is a peptide fragment produced by the cleavage of synaptic N-Methyl-D-aspartate receptors. The excitatory N-Methyl-D-aspartate receptor is one of the key regulators in the ischemic cascade of the brain. The NR2A/2B fragments can pass the blood-brain barrier and enter the bloodstream immediately after an episode of brain ischemia. These peptide fragments may act as foreign antigens and abnormally high concentrations initiate an immune response which generates measurable autoantibodies (aAbs) in the blood [18]. Both NR2A/B and NR2A/B aAbs are measured by enzyme-linked immunosorbent assay (ELISA); NR2A/B is also available as a point-of-care test.

NR2A/2B was tested as a single marker in a population of 192 patients suspected of ischemic stroke (IS diagnosis $n=101$, non-stroke diagnosis $n=91$ ). Dambinova et al. [19] reported a negative predictive value of $96.0 \%$ (95\% CI 92.3-98.3) and positive predictive value (PPV) of 93.0\% (95\% CI 86.1-97.1) for NR2A/2B applying the cutoff value of $1.0 \mu \mathrm{g} / \mathrm{L}$ in this population. Two previous studies of the same research group evaluating NR2A/2B aAbs were designed as case-control study. In the first study, NR2A/2B aAbs values did not differ significantly between 56 TIA and 31 ischemic stroke patients. A comparison with healthy volunteers resulted in a high C-statistic of 0.99 (no 95\% CI given) at an optimal cut-off value of $2.0 \mathrm{~g} / \mathrm{L}$ [14]. In the second study, interestingly, levels of NR2A/2B aAbs were higher in patients with prior TIA or stroke than in patients with a first acute TIA or stroke [20].

Parkinson 7, Nucleoside Diphosphate Kinase A, and Ubiquitin Fusion Degradation Protein-1

Parkinson 7 (PARK7), Nucleoside Diphosphate Kinase A (NDKA), and Ubiquitin Fusion Degradation protein 1, all 3 ELISA tests, were first identified in postmortem cerebrospinal fluid of stroke patients and later validated as early plasma markers of stroke by a research group from Switzerland. Allard et al. [21, 22] reported results of these markers in blood in 3 different cohorts, comparing stroke patients (total stroke $n=622$, TIA $n=$ $153)$ to healthy controls. Negative predictive values ranged from 57 to $92 \%$ and PPVs from 82 to $97 \%$, depending on the cut-off value applied [21,22]. Relevant to our review question is that these markers were equally increased in TIA patients and ischemic stroke patients. Also, all 3 markers seem to fit the clinically relevant time window, as biomarkers levels increased within $3 \mathrm{~h}$ after

Cerebrovasc Dis 2019;47:207-216 
Fig. 3. Forest plot with both reported and estimated AUCs per study, of candidate biomarkers that showed potential to be an early marker of TIA. $\mathbf{\square}$, cohort of suspected cases, reported AUC; $\square$, cohort of suspected cases, estimated AUC; $\boldsymbol{\Delta}$, case-control design, reported AUC; $\Delta$, case-control design, estimated AUC. AUC, area under the ROC curve; NR2A/B, N-methyl-D-aspartate receptor subunit; NR2A/B Abs, antibodies to NR2A/B; PARK-7, Parkinson 7; NDKA, nucleoside diphosphate kinase A; UFD-1, ubiquitin fusion degradation protein 1 ; H-FABP, heart-type fatty acid binding protein; $\mathrm{tPA}$, tissue plasminogen activator; PAI-1, plasminogen activator inhibitor 1; IL-6, interleukin-6; TSP, triage stroke panel.

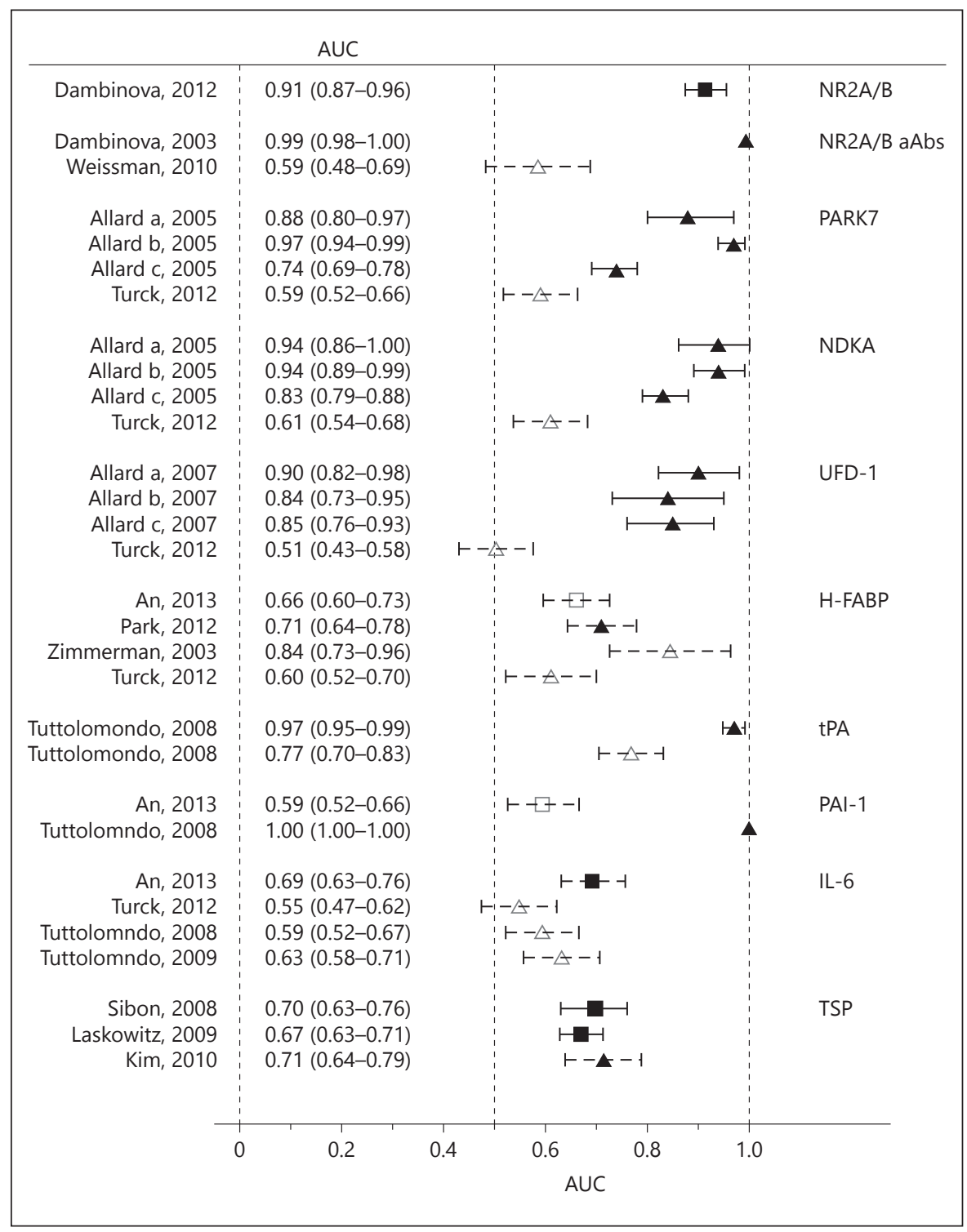

onset and did not differ from levels after $3 \mathrm{~h}$ (ranging till 5 days after onset).

A recent publication (2012) of the same research group showed an assessment of 29 biomarkers in a new cohort (103 strokes [19 TIAs] and 132 healthy controls). The main objective of this study was to determine if biomarkers can act as a time indicator, detecting very early stroke patients within the therapeutic window for thrombolysis. Accuracy data for differentiating strokes from healthy subjects are not given, but PARK7 and NDKA (and not ubiquitin fusion degradation protein-1) belong to the 5 markers that show the largest differences between cases and controls [23]. A study in a clinical population suspected of cerebrovascular disease is lacking.
Heart-Type Fatty Acid Binding Protein

Heart-type fatty acid binding protein (H-FABP) is a small protein involved in the intracellular transport and oxidation of fatty acids. It was named after its first detection in myocardium, but it is also enriched in neuronal cell bodies in the central nervous system, and is rapidly released from tissue to peripheral blood following an ischemic event. Besides a marker for cardiac ischemia, H-FABP has also been shown to be a marker for stroke [24]. Both H-FABP ELISA kits and point-of-care test are available.

All 4 studies identified that evaluated H-FABP found positive associations with ischemic stroke $[19,20,25,26]$. Two studies report accuracy measures, both from a case- 


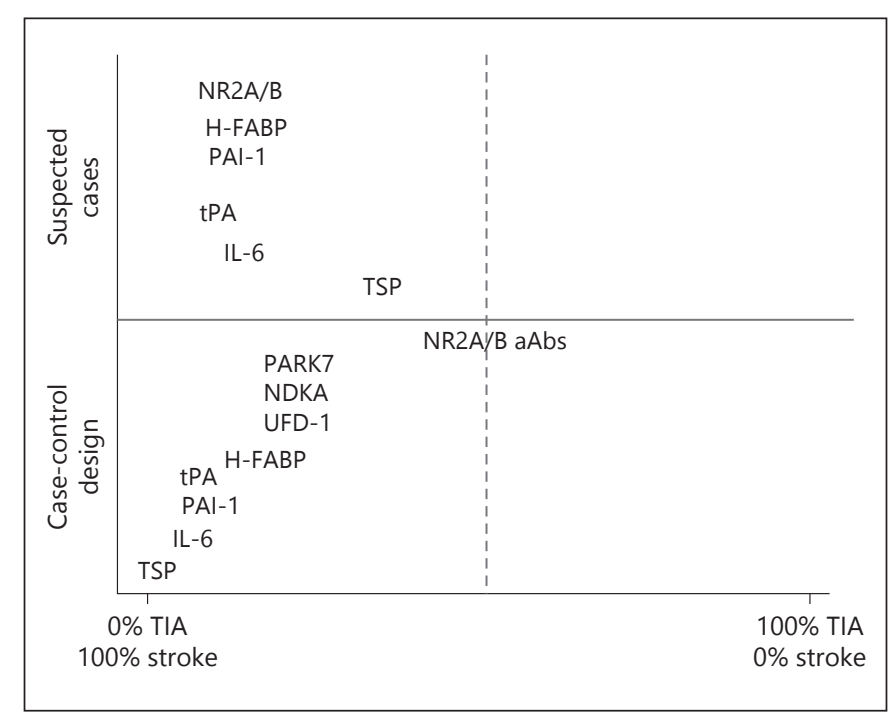

Fig. 4. Scoping plot depicting to what extent candidate markers have been evaluated in the domain of interest, that is, patients suspected of TIA. NR2A/B, N-Methyl-D-aspartate receptor subunit; NR2A/B aAbs, antibodies to NR2A/B; H-FABP, heart-type fatty acid binding protein; PAI-1, plasminogen activator inhibitor 1; tPA, tissue plasminogen activator; IL-6, interleukin-6; TSP, triage stroke panel; PARK-7, Parkinson 7; NDKA, nucleoside diphosphate kinase A; UFD-1, ubiquitin fusion degradation protein 1; TIA, transient ischemic attack.

control comparison with primarily strokes as cases. In 2004, a "pilot study" with a small sample size of 22 cases (11 IS, 6 ICH, 5 TIA) and 22 controls, reported 68.8\% sensitivity and $100 \%$ specificity [21]. However, in a larger population (111 ischemic strokes and 127 controls with other neurologic diagnoses), lower accuracy was found at a newly defined cut-off: $59.5 \%$ sensitivity and $79.5 \%$ specificity [20]. Based on these data, the authors conclude that $\mathrm{H}$-FABP appears to be unfit for use as a single marker because of limited sensitivity, but might add value in a panel of markers.

Tissue Plasminogen Activator and Plasminogen

Activator Inhibitor 1

Tissue plasminogen activator (tPA) and plasminogen activator inhibitor 1 (PAI-1), both measured by ELISA, are markers of thrombotic/fibrinolytic mechanisms. tPA is an enzyme involved in the breakdown of a blood clot by catalyzing the conversion of plasminogen to plasmin. We know recombinant tPA as thrombolytic drug in the early treatment of stroke. PAI-1 is a principal inhibitor of tPA.

Tuttolomondo et al. [27] first evaluated both markers in a case-control design with only ischemic stroke pa- tients $(n=120)$; the reported discriminative characteristics were remarkably high (AUC of tPA 0.97, and of PAI10.99 ).

Both markers have also shown diagnostic potency, however limited, in patients suspected of stroke or TIA. tPA was an independent predictor of stroke diagnosis (OR 1.63 [95\% CI 1.20-2.21] for the 75th vs. the 25 th centile of the marker distribution) in a study with 405 suspected stroke patients ( 40 of 285 strokes were TIAs) [28]. The authors also showed a modest yet non-significant improvement of the AUC by the addition of tPA to the Face Arm Speech Test (FAST; from 0.60 [95\% CI 0.550.65 ] to 0.66 [95\% CI 0.60-0.72]). PAI-1 was evaluated by An et al. [26] in addition to a clinical model in a population of 278 suspected strokes. PAI-1 was an independent predictor of stroke diagnosis in a model with age, sex, cardiovascular risk factors, and serum creatinine. However, PAI-1 did not remain as an independent variable in the best diagnostic model consisting of age, FAST, atrial fibrillation, and 3 other serum markers (S100B, MMP-9, and Interleukin-6 [IL-6]) [22].

\section{Interleukin-6}

IL-6 is a cytokine involved not only in inflammation and infection responses but also in the regulation of metabolic, regenerative, and neural processes. IL- 6 expression is increased in the brain following ischemia, and damaged neurons may contribute to its increased levels. IL-6 is one of the markers of inflammation most studied as stroke biomarker [29].

Various case-control studies demonstrate that plasma IL-6 is elevated in the acute phase of ischemic stroke [19, $23,30]$. Two studies have evaluated IL-6 in a clinical population of suspected stroke patients. In the previously mentioned Korean study by An et al. [26], IL-6 is among the 3 (out of 10) markers that are independent predictors in multivariate regression analyses including clinical variables (OR 1.77, 95\% CI 1.31-2.38, $p<0.001$ ). In this population with 175 ischemic stroke and 13 TIA patients, the panel IL-6/S100B/MMP-9 showed added value beyond age, atrial fibrillation, and FAST symptoms (AUC 0.865 vs. $0.837, p=0.069$ ) [22]. In the second study with 405 patients suspected of stroke, IL- 6 was associated with stroke diagnosis but had no added value beyond FAST in bivariate logistic regression analysis [24].

\section{Triage Stroke Panel}

The triage stroke panel (TSP) is a rapid, point-ofcare fluorescence immunoassay. It simultaneously measures 4 biomarkers (B-type natriuretic peptide, D-di- 
mer, matrix metalloproteinase-9, and S100B) resulting in a single composite result, the Multimarker Index (MMX) [16].

The MMX was developed by the BRAIN study group in a population of 1,146 patients suspected of stroke recruited at 17 different hospitals in the USA [15]. The model was created to discriminate between all stroke diagnoses (including TIA) and non-stroke diagnoses. Temporal validation was performed in a set of 343 patients recruited in the same hospitals after completion of the primary study, showing virtually identical discriminative characteristics. For all stroke diagnosis, the AUC was 0.69 (no 95\% CI given). The chosen optimal cut-off had a sensitivity of $90 \%$ and a specificity of $47 \%$. A sub-analysis showed that the discriminative capacity was poor for identifying TIA beyond $3 \mathrm{~h}$ after onset ( $0-3 \mathrm{~h}$; AUC 0.69 , 3-24 h; AUC 0.43-0.48).

The commercial TSP was evaluated in ED settings by 2 different research groups. Sibon et al. [17] found discriminative characteristics for all strokes (including TIA) comparable to the BRAIN study (AUC 0.70 [95\% CI $0.63-0.76$ ], sensitivity $94 \%$, specificity $24 \%$ ). Although there was no subgroup analysis for TIA (33 of 131 strokes), descriptive results show that the probability of TIA is virtually equal for MMX scores higher and lower than the MMX cut-off score of 1.3 [17]. Vanni et al. [16] evaluated TSP in 155 patients suspected of stroke, but they considered TIA as a non-stroke diagnosis in their analysis. Therefore, these results were not useful in answering our research question [16].

\section{MicroRNA}

MicroRNAs (miRNAs) are non-protein-coding short RNA molecules that regulate gene expression, and divided into intracellular and extracellular, or circulating, miRNAs [31]. The usefulness of miRNAs is now being evaluated for various diseases including ischemic stroke. Test methods for miRNA are more complex and part of an actively developing field. The studies on miRNAs we found with our search report high accuracy, but all had major methodological limitations [31-39]. Most studies generated new potential markers with a strategy of first selecting miRNAs with the largest difference between stroke and healthy subjects by miRNA profiling, and then presenting diagnostic accuracy for those miRNAs in the same patients. All studies had a case-control design, and validation studies were lacking. We identified an miRNA (miR-16) that showed increased serum expression levels in 2 different cohorts $[33,36]$.

\section{Discussion}

Our systematic literature review on blood biomarkers for the diagnosis of TIA shows that none of the biomarker studies evaluated the performance in our intended study population of interest that best reflects every day clinical practice: a population of patients (more or less) suspected of TIA by the clinician. There are studies providing accuracy data on detecting or excluding ischemic stroke, but most had methodological shortcomings. Small sample sizes, a case-control design comparing TIA(/ stroke) patients with healthy controls, data-derived thresholds, and not validating the performance externally in new patients all lead to a difficult to interpret and questionable evidence base for the role of these biomarkers in daily practice.

We identified a total of 124 different biomarkers being studied. They form a heterogeneous group of markers originating from various cell types and involved in very diverse cellular processes, many of which are not restricted to the brain. Some have a theoretical basis and were developed in animal or in vitro models, while others have been identified by comparing plasma or cerebrospinal fluid of stroke patients with that of healthy subjects.

Although evidence is limited, some markers might have added value beyond the clinical assessment in diagnosing brain ischemia, and specifically in TIA suspected cases. NR2A/B was the only single marker that had both high negative and PPVs in a population of suspected stroke ( $n=192$, with 101 ischemic strokes and 91 non-stroke diagnoses). Predictive values of PARK7 and NDKA were also high, but these data were all derived from case-control design studies thus overestimating the real diagnostic accuracy that should be calculated from the domain of suspected cases and then comparing cases with non-cases [5]. Other shortcomings are the small sample sizes of the separate cohorts and that different cut-offs were applied. Markers H-FABP, tPA, PAI-1, and IL-6 seem to be unfit as a single marker but may add value in a combination of markers. TSP, the first commercial panel of stroke markers, showed poor discriminative capacity in subgroups of suspected TIA patients. MiRNAs are a relatively new source of biomarkers and many new miRNAs are proposed by profiling studies, but to date the usefulness for TIA/stroke diagnosis remains uncertain because of the lack of reliable data.

Whiteley et al. [4] performed a systematic review of blood biomarkers in the diagnosis of ischemic stroke in 
2007. They similarly concluded that design and reporting of many biomarker studies was poor [4]. The 58 markers they identified largely correspond with the markers identified by our review as a result of the overlapping study domains. However, 10 years of research yielded many new proposed biomarkers and also new data on existing markers. Fifty-five of our 78 included studies were published after 2007.

The strength of our review is the clear focus on diagnostic markers for TIA, with a broad search as a starting point including studies that evaluated biomarkers for all-type brain ischemia. TIA and ischemic stroke are similar in that they are clinical expressions (of different degrees) of brain ischemia; thus, in principle, they largely share the same markers, except for markers of cell necrosis. A broad search was required to also identify biomarkers that showed potential as a diagnostic marker of ischemic stroke, but have not been evaluated for suspected TIA yet. The complete review process was performed by 2 reviewers who applied a modified QUADAS-2 tool to evaluate the quality of diagnostic studies.

Limitations must also be considered. We had to assess the performance of a large number of different markers in studies with much heterogeneity in design and reporting, and on average poor quality. It was therefore impossible to adequately compare the diagnostic potential of the various markers or even provide a summary odds ratio. The "selection" of the most promising markers applied in this review may irrespective of our specific criteria still hold some "residual" subjectivity. Considering the various utilities of biomarkers, we decided to narrow our search by a broad set of diagnostic terms as a filter because a validated diagnostic filter is lacking [40]. We may have missed potential biomarkers using this filter, although in our opinion it is unlikely that studies lacking our diagnostic terms would add biomarkers with supporting evidence of diagnostic value. Studies published after May 1, 2017, were not included in this review. A final concern is the possible effect of publication bias with underreporting of negative results.

A large study in patients suspected of TIA is needed to get a valid estimate of the accuracy of blood biomarkers. At present, it is doubtful whether a single marker would have add substantial diagnostic value beyond the clinical assessment. A multi-marker panel such as the TSP may produce higher accuracy, but combinations other than TSP need to be evaluated.

\section{Conclusions}

Currently, none of the evaluated biomarkers can be recommended for diagnosing TIA in suspected cases. Adequately performed diagnostic studies are needed that evaluate some of the promising markers in the domain of patients suspected of TIA.

\section{Acknowledgments}

None.

\section{Disclosures Statement}

None.

\section{Funding Sources}

None.

\section{References}

1 Giles MF, Rothwell PM. Risk of stroke early after transient ischaemic attack: a systematic review and meta-analysis. Lancet Neurol. 2007 Dec;6(12):1063-72.

2 Rothwell PM, Algra A, Chen Z, Diener HC, Norrving B, Mehta Z. Effects of aspirin on risk and severity of early recurrent stroke after transient ischaemic attack and ischaemic stroke: time-course analysis of randomised trials. Lancet. 2016 Jul;388(10042):365-75.

3 Kernagis DN, Laskowitz DT. Evolving role of biomarkers in acute cerebrovascular disease. Ann Neurol. 2012 Mar;71(3):289-303.
4 Whiteley W, Tseng MC, Sandercock P. Blood biomarkers in the diagnosis of ischemic stroke: a systematic review. Stroke. 2008 Oct; 39(10):2902-9.

5 Rutjes AW, Reitsma JB, Di Nisio M, Smidt N, van Rijn JC, Bossuyt PM. Evidence of bias and variation in diagnostic accuracy studies. CMAJ. 2006 Feb;174(4):469-76.

6 Liberati A, Altman DG, Tetzlaff J, Mulrow C, Gøtzsche PC, Ioannidis JP, et al. The PRISMA statement for reporting systematic reviews and meta-analyses of studies that evaluate health care interventions: explanation and elaboration. PLoS Med. 2009 Jul;6(7): e1000100.

7 Whiting PF, Rutjes AW, Westwood ME, Mallett S, Deeks JJ, Reitsma JB, et al.; QUADAS-2 Group. QUADAS-2: a revised tool for the quality assessment of diagnostic accuracy studies. Ann Intern Med. 2011 Oct;155(8):529-36.

8 Bertens LC, Broekhuizen BD, Naaktgeboren CA, Rutten FH, Hoes AW, van Mourik Y, et al. Use of expert panels to define the reference standard in diagnostic research: a systematic review of published methods and reporting. PLoS Med. 2013 Oct;10(10):e1001531. 
9 Easton JD, Saver JL, Albers GW, Alberts MJ, Chaturvedi S, Feldmann E, et al.; American Heart Association; American Stroke Association Stroke Council; Council on Cardiovascular Surgery and Anesthesia; Council on Cardiovascular Radiology and Intervention; Council on Cardiovascular Nursing; Interdisciplinary Council on Peripheral Vascular Disease. Definition and evaluation of transient ischemic attack: a scientific statement for healthcare professionals from the American Heart Association/American Stroke Association Stroke Council; Council on Cardiovascular Surgery and Anesthesia; Council on Cardiovascular Radiology and Intervention; Council on Cardiovascular Nursing; and the Interdisciplinary Council on Peripheral Vascular Disease. The American Academy of Neurology affirms the value of this statement as an educational tool for neurologists. Stroke. 2009 Jun;40(6):227693.

10 Moreau F, Asdaghi N, Modi J, Goyal M, Coutts SB. Magnetic Resonance Imaging versus Computed Tomography in Transient Ischemic Attack and Minor Stroke: The More You See the More You Know. Cerebrovasc Dis Extra. 2013 Oct;3(1):130-6.

11 Förster A, Gass A, Kern R, Ay H, Chatzikonstantinou A, Hennerici MG, et al. Brain imaging in patients with transient ischemic attack: a comparison of computed tomography and magnetic resonance imaging. Eur Neurol. 2012;67(3):136-41.

12 Faraggi D, Reiser B. Estimation of the area under the ROC curve. Stat Med. 2002 Oct; 21(20):3093-106.

13 Hanley JA, McNeil BJ. The meaning and use of the area under a receiver operating characteristic (ROC) curve. Radiology. 1982 Apr; 143(1):29-36.

14 Fon EA, Mackey A, Côté R, Wolfson C, McIlraith DM, Leclerc J, et al. Hemostatic markers in acute transient ischemic attacks. Stroke. $1994 \mathrm{Feb} ; 25(2): 282-6$.

15 Laskowitz DT, Kasner SE, Saver J, Remmel KS, Jauch EC; BRAIN Study Group. Clinical usefulness of a biomarker-based diagnostic test for acute stroke: the Biomarker Rapid Assessment in Ischemic Injury (BRAIN) study. Stroke. 2009 Jan;40(1):77-85.

16 Vanni S, Polidori G, Pepe G, Chiarlone M, Albani A, Pagnanelli A, et al. Use of biomarkers in triage of patients with suspected stroke. J Emerg Med. 2011 May;40(5):499-505.

17 Sibon I, Rouanet F, Meissner W, Orgogozo JM. Use of the Triage Stroke Panel in a neurologic emergency service. Am J Emerg Med. 2009 Jun;27(5):558-62.
18 Dambinova SA, Khounteev GA, Izykenova GA, Zavolokov IG, Ilyukhina AY, Skoromets AA. Blood test detecting autoantibodies to Nmethyl-D-aspartate neuroreceptors for evaluation of patients with transient ischemic attack and stroke. Clin Chem. 2003 Oct;49(10): 1752-62.

19 Dambinova SA, Bettermann K, Glynn T, Tews M, Olson D, Weissman JD, et al. Diagnostic potential of the NMDA receptor peptide assay for acute ischemic stroke. PLoS One. 2012;7(7):e42362.

20 Weissman JD, Khunteev GA, Heath R, Dambinova SA. NR2 antibodies: risk assessment of transient ischemic attack (TIA)/stroke in patients with history of isolated and multiple cerebrovascular events. J Neurol Sci. 2011 Jan; 300(1-2):97-102.

21 Allard L, Burkhard PR, Lescuyer P, Burgess JA, Walter N, Hochstrasser DF, et al. PARK7 and nucleoside diphosphate kinase $\mathrm{A}$ as plasma markers for the early diagnosis of stroke. Clin Chem. 2005 Nov;51(11):204351.

22 Allard L, Turck N, Burkhard PR, Walter N, Rosell A, Gex-Fabry M, et al. Ubiquitin fusion degradation protein 1 as a blood marker for the early diagnosis of ischemic stroke. Biomark Insights. 2007 Apr;2:155-64.

23 Turck N, Robin X, Walter N, Fouda C, Hainard A, Sztajzel R, et al. Blood glutathione $S$-transferase- $\pi$ as a time indicator of stroke onset. PLoS One. 2012;7(9):e43830.

24 Park SY, Kim MH, Kim OJ, Ahn HJ, Song JY, Jeong JY, et al. Plasma heart-type fatty acid binding protein level in acute ischemic stroke: comparative analysis with plasma S100B level for diagnosis of stroke and prediction of longterm clinical outcome. Clin Neurol Neurosurg. 2013 Apr;115(4):405-10.

25 Zimmermann-Ivol CG, Burkhard PR, Le Floch-Rohr J, Allard L, Hochstrasser DF, Sanchez JC. Fatty acid binding protein as a serum marker for the early diagnosis of stroke: a pilot study. Mol Cell Proteomics. 2004 Jan;3(1): 66-72.

26 An SA, Kim J, Kim OJ, Kim JK, Kim NK, Song $\mathrm{J}$, et al. Limited clinical value of multiple blood markers in the diagnosis of ischemic stroke. Clin Biochem. 2013 Jun;46(9):710-5.

27 Tuttolomondo A, Pinto A, Corrao S, Di Raimondo D, Fernandez P, Di Sciacca R, et al. Immuno-inflammatory and thrombotic/fibrinolytic variables associated with acute ischemic stroke diagnosis. Atherosclerosis. 2009 Apr;203(2):503-8.

28 Whiteley W, Wardlaw J, Dennis M, Lowe G, Rumley A, Sattar N, et al. Blood biomarkers for the diagnosis of acute cerebrovascular dis- eases: a prospective cohort study. Cerebrovasc Dis. 2011;32(2):141-7.

29 Scheller J, Chalaris A, Schmidt-Arras D, Rose-John S. The pro- and anti-inflammatory properties of the cytokine interleukin-6. Biochim Biophys Acta. 2011 May;1813(5):87888.

30 Lynch JR, Blessing R, White WD, Grocott HP, Newman MF, Laskowitz DT. Novel diagnostic test for acute stroke. Stroke. 2004 Jan; 35(1):57-63.

31 Long G, Wang F, Li H, Yin Z, Sandip C, Lou $\mathrm{Y}$, et al. Circulating miR-30a, miR-126 and let-7b as biomarker for ischemic stroke in humans. BMC Neurol. 2013 Nov;13(1):178.

32 Wang W, Sun G, Zhang L, Shi L, Zeng Y. Circulating microRNAs as novel potential biomarkers for early diagnosis of acute stroke in humans. J Stroke Cerebrovasc Dis. 2014 NovDec;23(10):2607-13.

$33 \mathrm{Wu} \mathrm{J}, \mathrm{Du} \mathrm{K}, \mathrm{Lu}$ X. Elevated expressions of serum miR-15a, miR-16, and miR-17-5p are associated with acute ischemic stroke. Int J Clin Exp Med. 2015 Nov;8(11):21071-9.

34 Jia L, Hao F, Wang W, Qu Y. Circulating miR145 is associated with plasma high-sensitivity $\mathrm{C}$-reactive protein in acute ischemic stroke patients. Cell Biochem Funct. 2015 Jul;33(5): 314-9.

35 Qian L, Yuanshao L, Wensi H, Yulei Z, Xiaoli C, Brian W, et al. Serum IL-33 Is a Novel Diagnostic and Prognostic Biomarker in Acute Ischemic Stroke. Aging Dis. 2016 Oct; 7(5): 614-22.

36 Tian C, Li Z, Yang Z, Huang Q, Liu J, Hong B. Plasma MicroRNA-16 Is a Biomarker for Diagnosis, Stratification, and Prognosis of $\mathrm{Hy}$ peracute Cerebral Infarction. PLoS One. 2016 Nov;11(11):e0166688.

37 Yang ZB, Li TB, Zhang Z, Ren KD, Zheng ZF, Peng J, et al. The Diagnostic Value of Circulating Brain-specific MicroRNAs for Ischemic Stroke. Intern Med. 2016;55(10):127986.

38 Zhao B, Zhu Z, Hao J, Wan Z, Guo X. Decreased plasma miR-335 expression in patients with acute ischemic stroke and its association with calmodulin expression. J Int Med Res. 2016 Dec;44(6):1331-8.

39 Chen Y, Song Y, Huang J, Qu M, Zhang Y, Geng J, et al. Increased Circulating Exosomal miRNA-223 Is Associated with Acute Ischemic Stroke. Front Neurol. 2017 Feb;8: 57.

40 Beynon R, Leeflang MM, McDonald S, Eisinga A, Mitchell RL, Whiting $P$, et al. Search strategies to identify diagnostic accuracy studies in MEDLINE and EMBASE. Cochrane Database Syst Rev. 2013 Sep;(9):MR000022. 